\title{
Kinetics and relaxation of electroresistance in transition metal oxides: Model for resistive switching
}

\author{
N. Das, ${ }^{1}$ S. Tsui,${ }^{1}$ Y. Y. Xue, ${ }^{1}$ Y. Q. Wang, ${ }^{1}$ and C. W. Chu ${ }^{1,2,3}$ \\ ${ }^{1}$ Department of Physics and TCSUH, University of Houston, Houston, Texas 77204-5002, USA \\ ${ }^{2}$ Hong Kong University of Science and Technology, Clear Water Bay, Kowloon, Hong Kong, China \\ ${ }^{3}$ Lawrence Berkeley National Laboratory, 1 Cyclotron Road, Berkeley, California 94720, USA
}

(Received 20 May 2009; published 10 September 2009)

\begin{abstract}
The kinetics of electric-field-induced resistive switching across metal ( $\mathrm{Ag}$ ) $-\mathrm{Pr}_{0.7} \mathrm{Ca}_{0.3} \mathrm{MnO}_{3}$ interfaces has been investigated. The resistance hysteresis $\Delta R$ varies with the pulse amplitude $V_{0}$ roughly as a step function with existence of a threshold voltage $V_{\mathrm{t}}$ for a fixed switching pulses width $T_{\mathrm{w}}$. On the other hand, the $\Delta R$ varies with the pulse width $\left(T_{\mathrm{w}}\right)$ as a two-stage sequence at a fixed $V_{0}$ : an initial exponential rise with a time constant $\tau_{\mathrm{S}} \approx 2 \times 10^{-7} \mathrm{~s}$ and a slow linearly increasing tail. The slow linear part is dominant only in the quasi-dc switch (pulse width $\sim$ a few seconds) below $V_{\mathrm{t}}$ but negligibly small above it. The retentions of the $\Delta R$ corresponding to the two stages are also extremely different, indicating that different underlying processes are involved. The relaxation time $\left(\tau_{\mathrm{R}}\right)$ is $10^{8} \mathrm{~s}$ ( $\sim$ year) or higher for the sub- $\mu$ s switching, in strong contrast with the total disappearance of the $\Delta R$ after a few days for subthreshold $\left(V_{0} \ll V_{\mathrm{t}}\right)$ quasi-dc switch. Different mechanisms, therefore, dominate the two different stages. More results obtained from time dependence study and impedance spectroscopy suggest that defect creation/annihilation, such as broken bonds under field, is likely the mechanism for the sub- $\mu$ s switching and that a slow accumulative process (like diffusion) of defects may be responsible for the subthreshold quasi-dc switch. Many observations further suggest that the accumulative process is much more complicated than simple migration/diffusion of the pre-existing defects.
\end{abstract}

DOI: 10.1103/PhysRevB.80.115411

PACS number(s): 73.40.-c, 71.55.-i, 72.80.Ga, 72.20.Jv

\section{INTRODUCTION}

\section{A. Background and motivation}

The search for nonvolatile resistive memory devices has been going on for quite a long time (almost a decade) now. Systems ranging from transition metal-oxide thin films [for example, perovskitelike $\mathrm{SrTiO}_{3}$ and $\mathrm{Pr}_{0.7} \mathrm{Ca}_{0.3} \mathrm{MnO}_{3}$ (PCMO) $]^{1-3}$ to binary oxides ${ }^{4}$ have been investigated. The exact mechanisms behind the room-temperature resistive switching across various oxide-electrode interfaces, however, remain elusive despite recent intense investigations. Various models, including carrier trapping and detrapping, ${ }^{1}$ defect creation annihilation by electric field, ${ }^{2}$ and change in doping level through electromigration, ${ }^{3,5}$ have been proposed. The models can first be roughly categorized as either based on pure electric processes or on processes involving lattice changes. As an example of the first category, Rozenberg et $a l .{ }^{1}$ tried to explain the switching as trapping/detrapping of injected carriers in pre-existing nanodomains adjacent to the metallic electrode. The charges trapped in turn modify the carrier transportation through these isolated islands (domains). Models belonging to the second category are more divergent, and may be further classified as migration/ diffusion of pre-existing defects, ${ }^{3,5}$ defect creation/recovery under high field, ${ }^{2}$ or heat-induced local phase transition. ${ }^{6}$ The situation may be further complicated by the fact that different samples (even with similar materials/configurations) show rather different switching/transport properties. ${ }^{7}$ For example, it has been demonstrated that oxygen electromigration may be responsible for the switching of single-crystal $\mathrm{SrTiO}_{3}$ under quasistatic I-V sweep. ${ }^{3}$ Similar migration/ diffusion of oxygen ion/vacancy, ${ }^{8,9}$ however, can hardly be accommodated with the nanosecond switching observed across metal-oxide interfaces. The well-established NernstEinstein (NE) relation ${ }^{7}$ challenges such possibility. Many probes have been used to explore the issue, but the picture is still unclear at this moment. Recent studies also have hinted ${ }^{7}$ that there might be no single unified mechanism even for the very limited cases of the metal-PCMO interfaces alone.

It is interesting to note that the switching kinetics and retention of switched states, i.e., their respective rates, should be closely related to the underlying mechanisms behind electroresistance. During any electronic trapping/detrapping process, for example, in the case of resistive switching, ${ }^{1}$ there is a competition between the trapping rate $\left(n_{\mathrm{loc}}+n_{\mathrm{hot}}\right) v \cdot n_{\mathrm{tr}} \sigma_{\mathrm{t}}$ and the release rate $n_{\mathrm{tr}} \cdot v_{0} \cdot e^{-\mathrm{U} / k \mathrm{~T}}$, where $n_{\mathrm{loc}}, n_{\mathrm{hot}}, n_{\mathrm{tr}}, v, \sigma_{\mathrm{t}}$, $v_{0}$, and $U$ are the concentrations of the thermal-equilibrium (local) carriers density, hot (injected) carriers density, traps density active during switching (may be a fraction of total traps available), carrier velocity, trapping cross section, a prefactor, and the trapping potential, respectively. Fast switching and good retention, e.g., qualities needed in flash memory devices, therefore, should constrain the related parameters involved in the above expressions. The migration/ diffusion, on the other hand, is an accumulative process across a macroscopic length. The resistance change during pulse switching under such mechanism, therefore, is expected to be proportional to the accumulative value of $\Sigma V_{0} \cdot T_{\mathrm{w}}$, where $V_{0}$ and $T_{\mathrm{w}}$ are the pulse amplitude and the pulse duration, respectively. A detailed investigation of the kinetics, therefore, should shed light on the underlying mechanisms.

Here we attempt to probe the topic from different directions. Voltage pulses with widths as narrow as $100 \mathrm{~ns}$ and as wide as 3 s, i.e., quasistatic I-V sweep, were used to switch metal- $\operatorname{Pr}_{0.7} \mathrm{Ca}_{0.3} \mathrm{MnO}_{3}$ samples. The retentions were mea- 


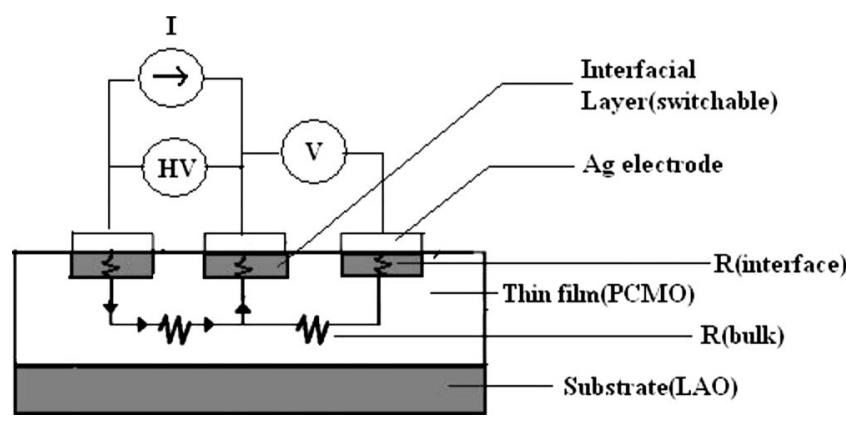

FIG. 1. A schematic diagram of the three-lead configuration used to measure the interfacial resistance and capacitance in the Ag-PCMO interface. $\mathrm{HV}$ is high voltage (to induce switch), $I$ is measurement current, and $V$ is interfacial voltage drop $(v)$.

sured during periods from a few hours to a few weeks. A close correlation between the switching voltage/duration and the relaxation rate of the metastable resistance states so formed is observed. Together with the impedance spectrum, we explored the main regions where various proposed models may play dominant roles. Based on our results a qualitative microscopic model has been developed.

\section{B. Experimental details}

The samples used here are PCMO thin films on $\mathrm{LaAlO}_{3}$ substrates $\left(10 \times 6 \mathrm{~mm}^{2}\right)$ with $\mathrm{Ag}$ electrodes deposited ex situ. The electrodes were rectangular with length $4 \mathrm{~mm}$ and width $0.5 \mathrm{~mm}$. The PCMO films (thickness $\approx 400 \mathrm{~nm}$ ) deposited through $a c$ sputtering are epitaxial with the preferred orientation based on the x-ray diffraction data. The substrate temperature for deposition was $800{ }^{\circ} \mathrm{C}$. A parallel threeelectrode configuration (Fig. 1), which removes the contribution from the bulk regions of the sample, is used to measure the interface resistance $R$ and capacitance $C .{ }^{10}$ The fast switching was induced through pulses with the pulse voltage $V_{0}$ up to $100 \mathrm{~V}$ and pulse width $T_{\mathrm{w}}$ down to $100 \mathrm{~ns}$. The pulses are generated by a DEI PVX-4150 function generator acting as a gate for a Keithley 2400 source meter. For slow switch (quasistatic de switch), a Keithley 2400 was used as a source. Switching voltage was on for $3 \mathrm{~s}$ (similar to pulse width of $3 \mathrm{~s}$ ) and then followed by a zero-bias $R$ measurement. The actual pulse voltage drop $V_{\mathrm{P}}$ across the interface layer, which is typically much smaller than the applied $V_{0}$, was measured using an HP54502A digital oscilloscope through the same three-lead configuration. Immediately after pulsing, a small measurement current $I(1 \mu \mathrm{A})$ was used to measure the interfacial $R$ with an HP34401A multimeter using the same three-lead configuration. The impedances were measured using an SR-830 lock-in amplifier (swept from $1-10^{5} \mathrm{~Hz}$ ) and a Solartron 1260 impedance analyzer (up to $10^{7} \mathrm{~Hz}$ ), again using the three-lead configuration with an excitation voltage of $100 \mathrm{mV}$. All of the retention studies were done with the Keithley 2400 source meter. For longtime retentions, (some data extend more than 3 days) extreme care has been taken to maintain a constant temperature. Although the retention studies were done in open air, the variation in the room temperature was also recorded.

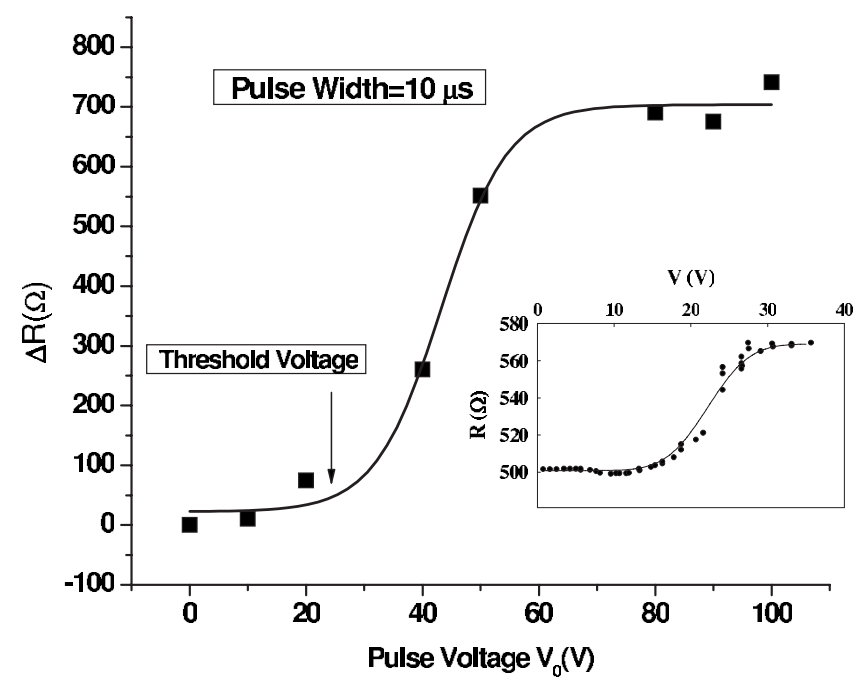

FIG. 2. Variation in resistance switch $(\Delta R)$ with pulse voltage $\left(V_{0}\right)$ for a fixed pulse width $\left(T_{\mathrm{w}}=10 \mu \mathrm{s}\right)$ in Ag-PCMO interface showing the existence of threshold voltage $\left(V_{\mathrm{t}}\right)$. Inset: variation of $R$ for a $10 \mu$ s positive pulse for another sample with different voltage threshold $\left(V_{\mathrm{t}}\right)$ but with similar characteristics.

Most of the temperature variation over time was negligible, but in some cases a base subtraction has been done.

\section{RESULTS AND DISCUSSION}

\section{A. Kinetics of switch}

\section{Submicrosecond pulse switching}

We began with a conventional resistive switch in metal (Ag)-PCMO interface induced by a fast electric pulse. The resistance of the interface has been altered successfully between stable high resistance state (HRS) and low resistance state (LRS), with switching time as short as 100 ns. The switch obtained here is bipolar in nature with positive voltage (current going into Ag electrode and film) producing HRS, and the resistance split $\Delta R=R_{\mathrm{HRS}}-R_{\mathrm{LRS}}$ was recorded by two sequential pulses with alternating polarities. Figure 2 represents the $\Delta R$ variation with switching (pulse) voltage $\left(V_{0}\right)$ for a fixed pulse width $T_{\mathrm{w}}=10 \mu \mathrm{s}$ on an Ag-PCMO sample (sample A). The data can be fitted to a sigmoidlike curve with a threshold voltage $V_{\mathrm{t}}(\sim 25 \mathrm{~V})$ below which the $\Delta R$ is negligibly small. There also exists an upper plateau for higher voltages. While the exact threshold voltage value changes from sample to sample, nature off the plot is universal. For example, variation in virgin interfacial resistance $(R)$ from another sample after $10 \mu$ s positive pulse with increasing voltage has been shown in the inset of Fig. 2 . The resistance variation exactly mimics the variation of $\Delta R$ shown in Fig. 2, confirming the existence of intrinsic threshold barrier. The pulse voltage range above $V_{\mathrm{t}}$ is usually very limited, and the interfacial layer is typically burned at pulse voltage of a few multiples of $V_{\mathrm{t}}$. The $V_{\mathrm{t}}$ value in a given sample, however, seems to be insensitive to $T_{\mathrm{w}}$ in the range $100 \mathrm{~ns}-10 \mu \mathrm{s}$. The existence of such a threshold suggests the presence of a microscopic energy barrier ${ }^{2,10}$ instead of an accumulative and threshold-less process such as atomic/ 


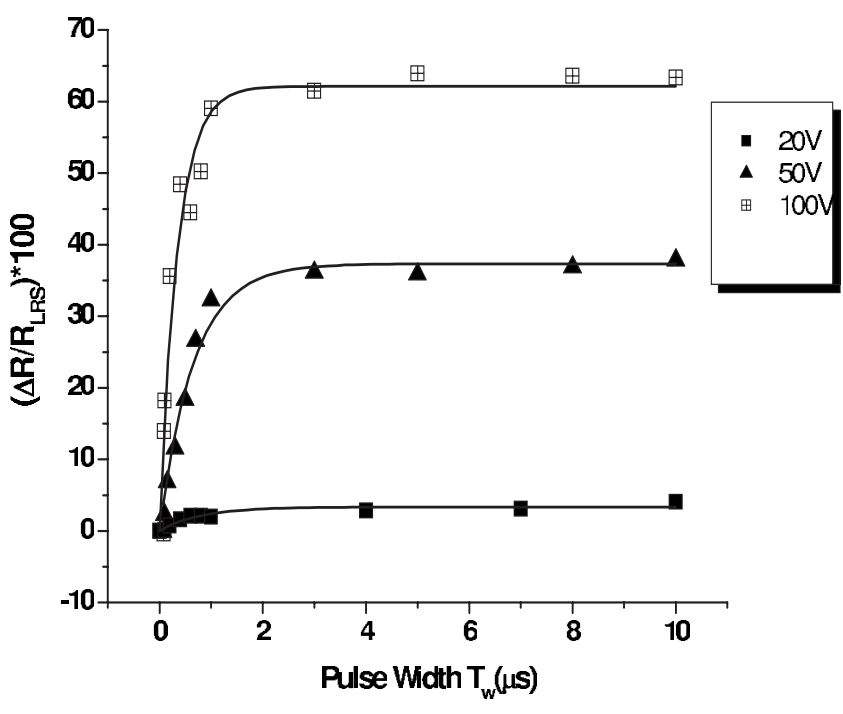

FIG. 3. Plot of percentage change in the low resistance state $\left(R_{\mathrm{LRS}}\right)$ with pulse width $\left(T_{\mathrm{w}}\right)$ for three different pulse voltages $\left(V_{0}\right)$. Two distinct regions (an exponential rise followed by a plateau) can be clearly identified.

vacancy migration. ${ }^{5}$ We next turned our attention to the $T_{\mathrm{w}}$ dependency of the $\Delta R$ at fixed $V_{0}$. The switching speed, to our surprise, appears to be totally different below and above $V_{\mathrm{t}}$. Figure 3 shows a percentage change in resistance $\left\{\left[\left(R_{\mathrm{HRS}}-R_{\mathrm{LRS}}\right) / R_{\mathrm{LRS}}\right]^{*} 100\right\}$ with pulse width $T_{\mathrm{w}}$ (sample A) for different $V_{0}(100,50$, and $20 \mathrm{~V})$, both far above and close to its voltage threshold $V_{\mathrm{t}}(\approx 25 \mathrm{~V})$. The dependency on the $T_{\mathrm{w}}$ can be clearly separated into two parts: an exponential rise (with a time constant $\tau_{\mathrm{S}} \sim 200 \mathrm{~ns}$ ) and a plateau after $1-2 \mu \mathrm{s}$. It is especially interesting that the time constant of the exponentially rising part is almost independent of the pulse amplitude. All the above observations showing a fast transitions, such as behavior, are in disagreement with the general idea that either an accumulated process or simple thermal-assisted tunneling ${ }^{3,5}$ can explain fast switching simply due to the fact that the nature of evolution of switch changes heavily with voltage range. Below $V_{\mathrm{t}}$, however, not only is the fast sub- $\mu$ s stage largely suppressed, but the plateaus after $1-2 \mu$ s are replaced by a slow quasilinear rise with $T_{\mathrm{w}}$ (Fig. 4). In Fig. 4, we have plotted a subthreshold switch $\left(V_{0}<V_{\mathrm{t}}\right)$ for $T_{\mathrm{w}}=10 \mu$ s with two different pulse voltages $\left(V_{0}=10\right.$ and $\left.20 \mathrm{~V}\right)$. A significant contribution coming from some accumulated process, similar to the proposed diffusion/migration, ${ }^{3,8,9}$ can be predicted based on the continuous quasilinearity of those plots. It should be pointed out that different samples show rather divergent $V_{\mathrm{t}}$. The observed $V_{\mathrm{t}}$, for example, varies from as low as $1 \mathrm{~V}$ and up to $100 \mathrm{~V}$, the upper limit of the setup. Such subthreshold switching, however, appears to share rather common features. Not only are the relative contributions from two different regions (fast exponentially rising and linear plateau) insensitive to the $V_{0}$ (as long as $V_{0}<V_{\mathrm{t}}$ ) for a given sample (Fig. 4), but the scaled $\Delta R / \Delta R(10 \mu \mathrm{s})$ for subthresholds switch in case of two different samples are also comparable (inset of Fig. 4, each symbol represent a different sample). While this may be a coincidence, a significant contribution from the accumulative part below $V_{\mathrm{t}}$ is a well-established fact for all samples examined.

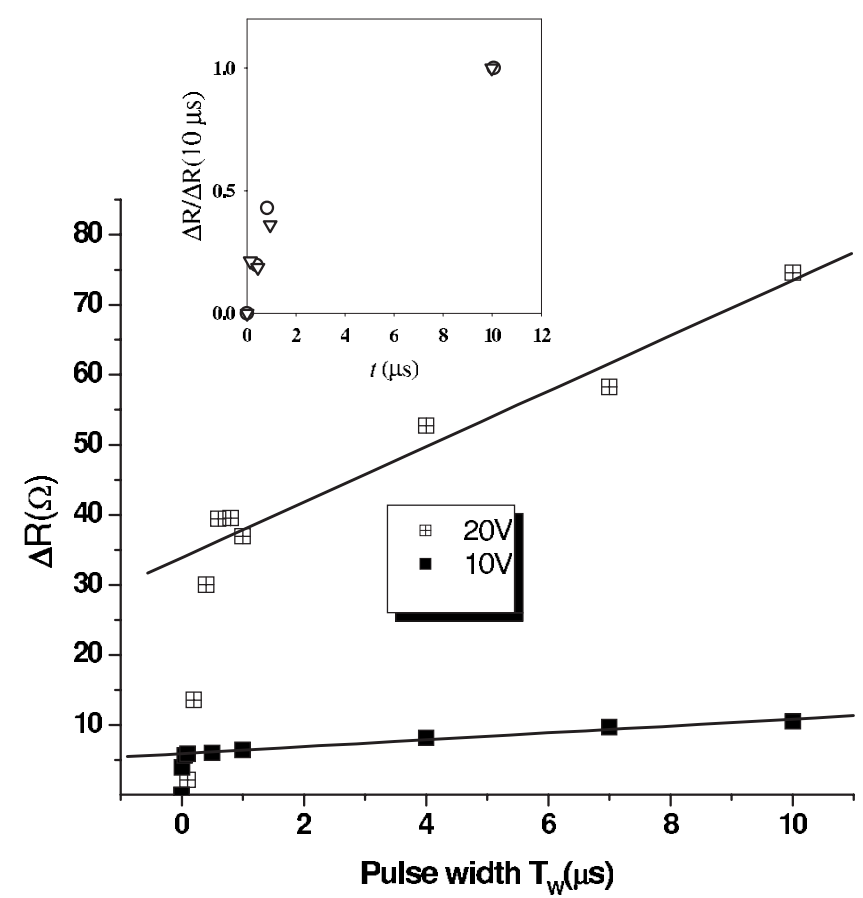

FIG. 4. Subthreshold resistance switching in Ag-PCMO interface. The variation in resistance switch $(\Delta R)$ with pulse width $\left(T_{\mathrm{w}}\right)$ for two different subthreshold pulse voltages $\left(V_{0}<V_{\mathrm{t}}\right)$. Inset: a normalized subthreshold $\left(V_{0}<V_{\mathrm{t}}\right)$ resistance switch $\left[\Delta R / \Delta R\left(T_{\mathrm{w}}\right.\right.$ $=10 \mu \mathrm{s}$ )] plotted for two different samples (open circles and open triangles), showing the universality of the plot.

\section{Slow quasistatic dc switch}

It is interesting to note that switching can even be observed under quasistatic I-V sweeps for the samples never showing noticeable switching under 1-2 $\mu$ s pulses up to $100 \mathrm{~V}^{7}$ Sample B, for example, has shown no noticeable switching with $T_{\mathrm{w}} \leq 10 \mu \mathrm{s}$ and $V_{0} \leq 100 \mathrm{~V}$, i.e., a horizontal $\Delta R\left(T_{\mathrm{W}}\right)=0$ line in a $\Delta R$ vs $T_{\mathrm{W}}$ plot similar to Fig. 4 . It, however, shows considerable $R$ hysteresis even below $20 \mathrm{~V}$ if a series of pulses with varying amplitude but with a fixed width $T_{\mathrm{w}}=3 \mathrm{~s}$ is applied to simulate a quasistatic I-V sweep, i.e., with the same polarity but increasing (decreasing) amplitude in an individual branch (Fig. 5). A small measurement current of $1 \mu \mathrm{A}$ was used to check the resistance change after each pulse. The hysteresis loop, however, looks different: the $R$ "jumps" may occur not on the amplitudeincrease branch $\left[V_{0}=0 \rightarrow V_{\max }(+)\right]$ as expected, but rather in the middle of the amplitude-decrease branch $\left[V_{0}=V_{\max }(+)\right.$ $\rightarrow 0]$, i.e., the pulse amplitude may not be the only dominant factor. $V_{\max }(=20 \mathrm{~V})$ is the maximum switching voltage value in the I-V sweep (still $V_{\max }<V_{\mathrm{t}}$, making it a subthreshold switch). Similar situations have been observed by us and others. ${ }^{11-13}$ Such strange behavior, however, can be easily understood if the resistance data are replotted against the accumulated effect of applied voltage $\left(V_{0}\right)$ over total pulse time given by $\Sigma V_{0 i} T_{0 i}\left(=T_{w} \Sigma V_{0 i}, T_{\mathrm{w}}\right.$ being a constant throughout the switch) for the entire I-V sweep (Fig. 6). The hysteresis between the $V_{0}$ increase and the $V_{0}$ decrease branches becomes relatively minor but still noticeable, and the $R$ "jump" between LRS and HRS mainly an accumula- 


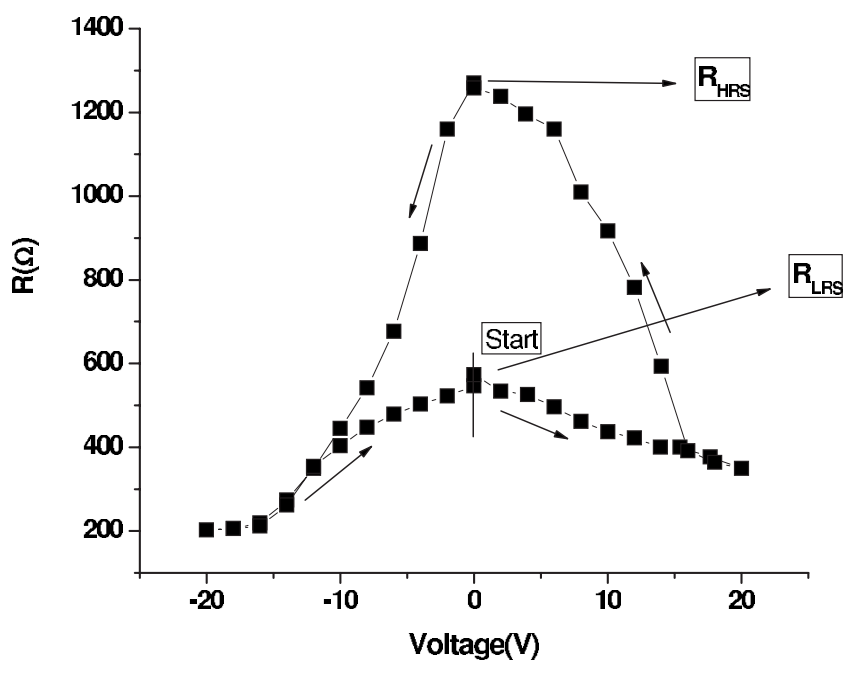

FIG. 5. (a) Quasistatic I-V sweep producing slow switch in PCMO-Ag interface. The data obtained by applying a $3 \mathrm{~s}$ voltage pulse followed by a resistance measurement and then repeating the process as voltage is ramped up.

tive, though rather rapid nonlinear evolution with $\Sigma V_{0 i} T_{w i}$. The metastable resistances at the HRS and LRS can survive long enough to allow the relaxation being measured, could simply result in a much smaller probe current. Although this is rather similar to the electromigration process previously proposed, the data show significant differences: the resistance actually varies with $\Sigma V_{0 i} T_{w i}$ nonmonotonically. The initial $R_{\text {LRS }}$ even decreases significantly with the accumulated "charge" below $\Sigma V_{0 i} T_{w i}=100 \mathrm{~V} \mathrm{~s}$, keeps almost a constant between 200 and $400 \mathrm{~V} \mathrm{~s}$, and "switches" to the higher $R_{\mathrm{HRS}}$ only in range $450-600 \mathrm{~V} \mathrm{~s}$. This is in great contrast with a pure accumulative process, e.g., various migration/ doping-level models. A linear dependency is expected if the

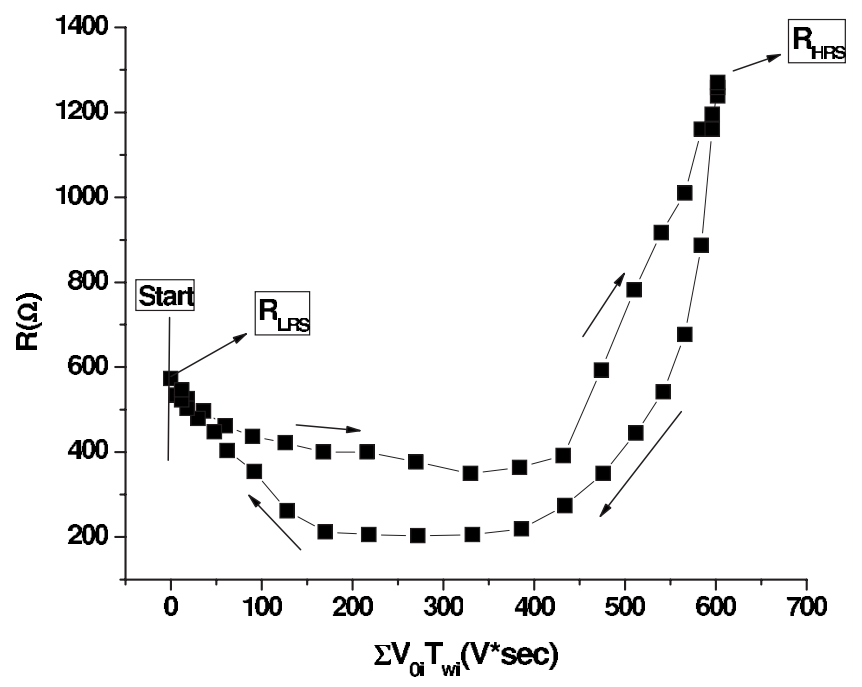

FIG. 6. The accumulative nature of slow subthreshold switch in Ag-PCMO switch. $R(\Omega)$ has been plotted against the accumulative value of $\Sigma V_{0 i} T_{\mathrm{wi}}\left(=T_{\mathrm{w}} \sum V_{0 i}, T_{\mathrm{w}}\right.$ is constant, and $3 \mathrm{~s}$ pulse width). The summation has been evaluated for all four voltage branches shown in Fig. 5 (i.e., $V_{0}$ varies from $0 \rightarrow V_{\max }(+), V_{\max }(+) \rightarrow 0,0$ $\rightarrow V_{\max }(-)$, and $V_{\text {max }}(-) \rightarrow 0$, where $V_{\max }=20 \mathrm{~V}$ ).

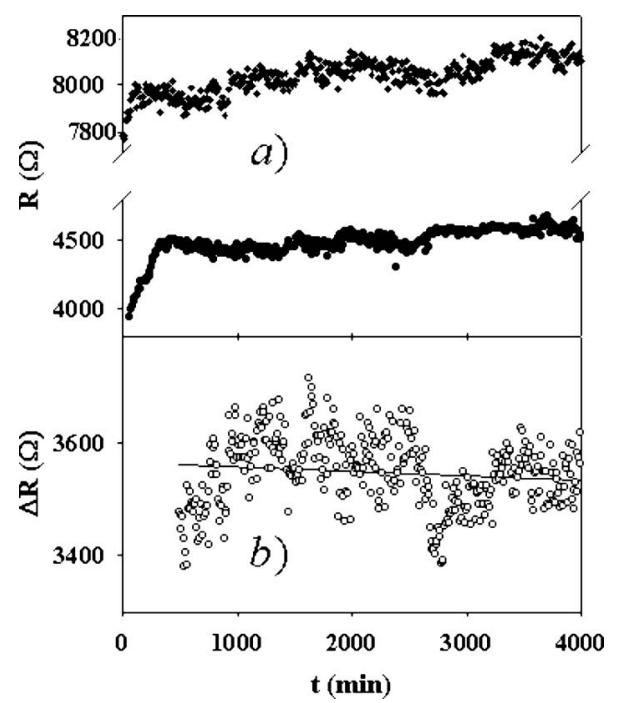

FIG. 7. (a) Time dependence and stability of $R$ ( $R_{\mathrm{HRS}}$ : solid diamonds and $R_{\mathrm{LRS}}$ : solid circles) for sub- $\mu$ s fast switch in $\mathrm{Ag}$ PCMO interface for a few days. Most of the relaxation is limited to a small-time window $(t=500 \mathrm{~min})$. (b) Variation of $\Delta R$ for a longer-time window. With some data fluctuation, the time period of relaxation is at least $\sim 10^{8} \mathrm{~s}(\sim$ years $)$, making it a very stable switch.

resistance is proportional to the change in doping level caused by the electromigration ${ }^{5}$ or monotonic if the situation is more complicated. The above results are rather different from that found in bulk $\mathrm{SrTiO}_{3} \cdot{ }^{3}$ Effect from multiple competing processes must be included to explain such trends, which will be treated in length later. The nonlinearity caused by the space charges of carriers, for example, may lead to the initial (reversible) $R$ decrease with $|V|$ in Fig. 5.

\section{B. Relaxation studies}

From the perspective of potential device application, stability of resistive switched states is one of the main issues. In addition, the nature of relaxation of the switched states should also shed some light on the nature of the process itself. As discussed above, the switching triggered by the sub- $\mu$ s pulses and the quasistatic I-V sweep might be completely different processes. We therefore explored them separately. A typical relaxation data set after a $100 \mathrm{~V}, 1 \mu$ s pulse has been presented in Fig. 7 (sample C). There are two clear features immediately observable. First, both the $R_{\mathrm{HRS}}$ and the $R_{\mathrm{LRS}}$ (represented by solid diamonds and solid circles, respectively) show two distinguishable relaxation regions: one below 200-500 min and another after 500 min [Fig. 7(a)]. Similar behavior, i.e., noticeable relaxations mainly occur before the first $500 \mathrm{~min}$, has been observed in all samples we investigated. Second, the split $\Delta R=R_{\mathrm{HRS}}-R_{\mathrm{LRS}}$ after $500 \mathrm{~min}$ is nearly relaxationless [Fig. 7(b)]. Despite the data fluctuations, the $\Delta R$ between 500 and $4000 \mathrm{~min}$ can be fit as an exponential decay function $\Delta R_{0} e^{-\left(t / \tau_{R}\right)}$ of the time $t$. The time constant $\tau_{\mathrm{R}}=(3.6 \pm 1) \times 10^{8} \mathrm{~s}$ suggests a retention time of a year, in reasonable agreement with previous reports. ${ }^{8}$ It confirms that such sub- $\mu$ s switching has an extremely large ratio 


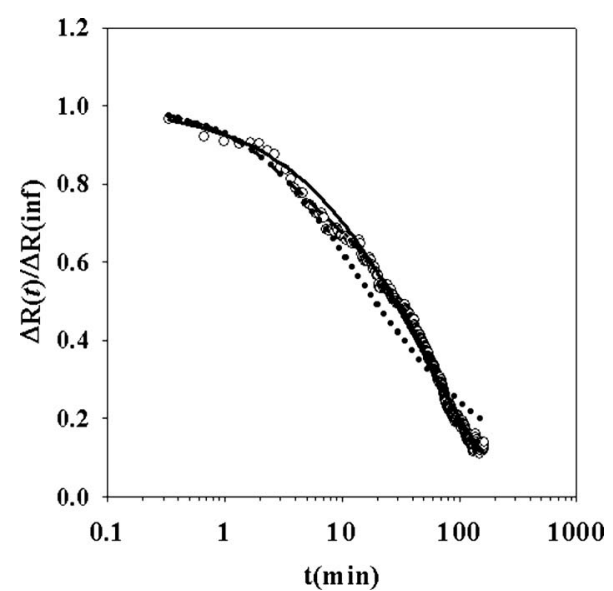

FIG. 8. Relaxation in short-time window $(t=0 \rightarrow 200 \mathrm{~min})$ has been fitted with different existing relaxation mechanisms. Open circles: raw data; solid line: stretched exponential relaxation; dotted line: diffusion model; and dashed line: sum of two exponential decay terms.

of $\tau_{\mathrm{R}} / \tau_{\mathrm{S}}$ on the order of $10^{15}\left(\tau_{\mathrm{S}}\right.$ is fast switching time $\sim 2$ $\times 10^{-7} \mathrm{~s}$ ), which is far larger than that allowed for migration/diffusion models under the NE constraint. ${ }^{7}$ It is also interesting to note that such a slow rate of decay (long retention time) will imply an energy barrier on the order of $k \mathrm{~T} \ln \left[v_{0} . \tau_{R}\right] \approx \mathrm{a}$ few $\mathrm{eV}$, a value more suitable for the process of bonds breaking, ${ }^{14}$ where $v_{0} \approx 10^{10} / \mathrm{s}$ is the characteristic frequency of ion oscillations. Although the exact nature of the bond breaking is not known, the energy of the hot carriers injected from the metal electrode is enough to cause such lattice damage above $V_{\mathrm{t}}{ }^{15,16}$

The relaxations occurring in the first $500 \mathrm{~min}$ are worth a closer look. The first unusual characteristic is that both the $R_{\text {HRS }}$ and the $R_{\text {LRS }}$ shift to higher values with $t$ [Fig. 7(a)]. This happens in most cases investigated here. Together with the fact that both are typically much higher than that of the virgin sample, the relaxation seems to point toward a common metastable state rather than the equilibrium oxygen stoichiometry of the experimental virgin conditions. The trend also challenges the models that regard the switching merely as a field-driven donor redistribution. ${ }^{1}$ Any given deviation in carrier concentration from its metastable virgin-state value should be suppressed during the relaxation processes if its gradient is the only driven force for the relaxation. The resistance at the disturbed state, therefore, should decrease during the relaxation. To further explore the puzzle, the $t$ dependency of the $\Delta R$ after a $50 \mathrm{~V}, 10 \mu$ s pulse was studied (Fig. 8). The $R$ at $500 \mathrm{~min}$ is used as the quasiequilibrium resistance [beyond $500 \mathrm{~min}$ the resistance states have been very stable with very little relaxation, justifying the definition $\Delta R$ (inf) used] and the normalized split $\Delta R_{\mathrm{N}}$ $=[R(500 \min )-R(\mathrm{t}) / R(500 \min )-R(0)]=\Delta R(\mathrm{t}) / \Delta R($ inf $) \quad$ is used for comparisons with different relaxation models. We compare our data with a general stretched exponential relaxation phenomena found in disordered materials ${ }^{17-19}$ given by $\Delta R_{\mathrm{N}}=e^{-(t / \tau)^{\alpha}}$, where $\tau$ and $\alpha(<1)$ are the time constant and the characteristic index, respectively. Although the origin of such relaxation behavior can be quite complicated and di- verse, a general consensus regarding the role of lattice imperfections (defect, vacancy, dangling bonds, and many more) as one of the major contributor has been established. In this present study, we do not have the scope to go microscopic detail calculations regarding the evolution of such time dependence but will merely borrow the functional from to identify underlying switching process. Keeping in mind the proposed diffusion model for switching, ${ }^{5}$ attempts has also been made to fit the relaxation databased on the general one-dimensional diffusion equation for thin films, ${ }^{20}$ where the concentration $C(x, t)$ of the unbalanced defects can be written as a convolution of the initial distribution of $M\left(x_{0}\right)$ and the Green's function $\frac{e^{-\left[\left(x-x_{0}\right)^{2}\right] / 4 D t}}{\sqrt{\pi D t}}$ of the diffusion equation, where $D$ is the diffusion constant. Assuming a linear relationship between $C(x, t)$ and $R(t)$ as well as a Gauss-like $M\left(x_{0}\right)$, a square-root dependency, i.e., $\Delta R_{\mathrm{N}} \propto \frac{1}{\sqrt{1+4 \frac{D}{L^{2}}}} \propto \frac{1}{\sqrt{t_{0}+t}}$, was expected for long-range diffusion, ${ }^{5}$ where $L$ is the length scale (width) of the initial Gauss distribution and $t_{0}$ is a fitting parameter. Both models can fit the data well with the moderate run-to-run resolution, i.e., they cannot be used to single out a unique process responsible for the switching mechanism (Fig. 8). It is interesting to note that a sum of two exponential relaxations ${ }^{21}$ can fit the data even better. In Fig. 8 , open circles represent raw data, solid line for stretched exponential relaxation fitting, dotted line for diffusion model fitting, and dashed line represents the fitting of sum of two exponential decay terms. Realizing that any defect-creating process should unavoidably slightly distort surrounding lattices, a rearrangement of surrounding ions/atoms, i.e., either a stretched exponential decay or a sum of several simple exponential decays, seems to be a more reasonable interpretation here. For verification, the relaxations at different pulse widths $\left(T_{\mathrm{w}}\right)$ were compared. The thickness $L\left(\propto t_{0}\right)$ of the disturbed layer in the diffusion model is expected to increase with $T_{\mathrm{w}}$ (accumulative time dependence) before the $\Delta R$ is saturated, i.e., $t_{0}$ should increase with $T_{\mathrm{w}}$. Pulse widths of $100 \mathrm{~ns}, 1 \mu \mathrm{s}$, and $10 \mu \mathrm{s}$ (pulse voltage $V=50 \mathrm{~V}$ ) were then used (Fig. 9). The initial unrelaxed switched resistance at $t$ $=0$ strongly depends on $T_{\mathrm{w}}$, demonstrating that the disturbance is far from saturation [Fig. 9(a)]. The observed relaxation of $R_{\mathrm{LRS}}$, however, is independent of $T_{\mathrm{w}}$ [Fig. 9(b)]. Even the total amount relaxed (can be roughly calculated from Fig. 9(b), i.e., $[R(t)-R(500 \mathrm{~min})])$ is $T_{\mathrm{w}}$ independent (repeated experimental run do show some variation but extremely small compared to total value) despite the fact that the total $R_{\text {LRS }}$ (and corresponding $\Delta R$ ) varies more than two times. This is in strong disagreement with the diffusionoriented mechanisms. Therefore, fast-relaxation part of the switching invoked by overthreshold $\left(V_{0}>V_{\mathrm{t}}\right)$ sub- $\mu$ s pulses is not associated with an accumulative process such as diffusion. It is also interesting to note that the unrelaxed $R_{\mathrm{LRS}}$ actually decreases with $T_{\mathrm{w}}$ [Fig. 9(a), open circle], a trend in disagreement with any accumulative interpretations based on virgin oxygen stoichiometry but in agreement with the fact that both $R_{\mathrm{HRS}}$ and $R_{\mathrm{LRS}}$ relax to higher resistances (Fig. 7) as discussed earlier. We therefore suggest that the first-stage relaxation is the result of recovery of surrounding lattice distortions, which are the unavoidable by-products of any bond 


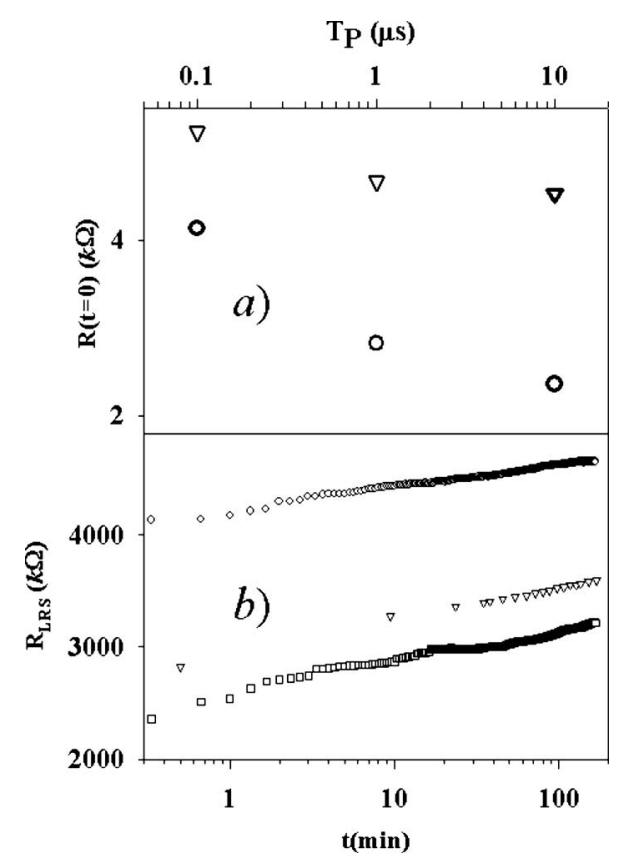

FIG. 9. (a) Change in initial unrelaxed resistance $(R, t=0)$ of Ag-PCMO interface with different pulse width $\left(T_{\mathrm{W}}\right)$ plotted in log scale (base 10). Open triangles: $R_{\mathrm{HRS}}$; open circles: $R_{\mathrm{LRS}}$. (b) Relaxation of $R_{\mathrm{LRS}}$ for three different pulse widths has been plotted, showing the pulse-width-independent behavior of resistance relaxation. Open circles: $100 \mathrm{~ns}$; open triangles: $1 \mu \mathrm{s}$; and open squares: $10 \mu \mathrm{s}$. Pulse voltage $V=50 \mathrm{~V}$.

breaking. It is interesting to note that even if unrelaxed, $R_{\mathrm{HRS}}$ and $R_{\mathrm{LHS}}$ decrease but $\Delta R$ still increases with $T_{\mathrm{W}}$ and consistent with the data in Fig. 3.

In estimating the associated barrier height, it is interesting to note that the stretched exponential decay is actually the result of the convolution of the potential distribution, ${ }^{22}$ and the correlation $\Gamma=\nu_{0}^{-1} e^{E_{\tau} / k T}$ may still be roughly held, where $\Gamma, E_{\mathrm{r}}$, and $v_{0}$ are the relaxation time, the barrier height, and the characteristic frequency, respectively. With $v_{0}$ $\left(10^{10}-10^{11} \mathrm{~Hz}\right)$, the barrier height corresponding to the above time window (for $\Gamma$ ) of $10^{1}-10^{4} \mathrm{~s}$ is $0.6-0.7 \mathrm{eV}$ or higher. Typically, diffusion of point defects (Schottky defects, etc.) in crystalline solids or Si involve barrier potentials $\ll 0.6 \mathrm{eV} .{ }^{23,24}$ Even lower barriers have been proposed for the defect diffusion across the metal-oxide interfaces to accommodate the fast switching observed, which clearly does not support relaxation data. The estimated barrier height for the relaxation, therefore, certainly does not favor a migration/diffusion model supporting our earlier conclusion.

We next turned our attention to slow quasistatic switch presented in Fig. 5 and study their relaxation behavior. We studied the retention behavior for both $R_{\mathrm{RHS}}$ and $R_{\mathrm{LHS}}$ (inset, Fig. 10) over a few days. It is interesting to note that the relaxation is totally different from those after sub- $\mu$ s pulses; most of the $\Delta R$ is relaxed within $20 \mathrm{~h}$ and the $R$ hysteresis is completely gone after a few days. Another interesting issue in the data is the relaxation direction: both $R_{\mathrm{HRS}}$ and $R_{\mathrm{LRS}}$ increase with $t$ although the sweeps actually change the lower virgin-state resistance to a higher value (Fig. 10). Two repeated measurements are plotted in Fig. 10. The run-to-run

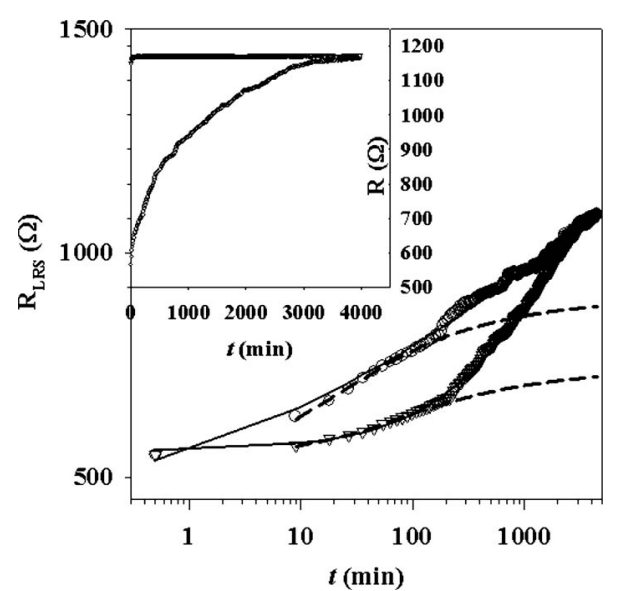

FIG. 10. Variation of $R_{\mathrm{LRS}}$ with time and fitting of different possible relaxation mechanisms. Two different data sets (open circles and open triangles) have been plotted for the same sample showing the universality of the plot in spite of run-to-run variation. Solid line: stretched exponential relaxation fit; dotted line: 1D diffusion equation fit. Inset: retention for slow subthreshold switch in Ag-PCMO interface (Fig. 5). The switch completely disappears within a few days as $R_{\mathrm{LRS}}$ relaxes back to $R_{\mathrm{HRS}}$.

change is significant. The $t$ dependency is again compared with the stretched exponential decay and the expected defect diffusion. While the stretched exponential decay (solid lines) fits both data sets rather well, the diffusion model (dashed lines) can only reasonably fit the initial 60-120 min data. The data in the broader time window significantly deviated from that expected from the diffusion models. Actually, the modulus of apparent slope given by $\left|\frac{d}{d(\ln t)}(R)\right| \propto \mid t \cdot \frac{d}{d t}\left(t_{0}\right.$ $+t)^{-1 / 2}$ is expected to decrease with $t$ in the diffusion model and in disagreement with the accelerated rate above $200 \mathrm{~min}$ (Fig. 10). Although a single diffusion constant $D$ cannot be fitted properly with the whole data range in Fig. 10, but the data can be fitted piecewise, if we assume the existence of more than one diffusion process [with different $D$, very similar to double exponential fitting in fast switching (Fig. 9)]. It should be noted that to distinguish the results from stretched exponential decay and/or that expected by diffusion mechanisms is very difficult by the $t$ dependency alone and other techniques are needed to help resolve the issue. For example, in the case of the slow switch, although a longer-time window seems to favor the stretched exponential model, in the following sections, we will show theoretically (NE correlation) and experimentally (by impedance spectroscopy) that accumulative/diffusion model is more suitable candidate for slow subthreshold switch. The mechanisms responding for the quasi-dc switching, therefore, should be more complicated than simple migration/diffusion. ${ }^{5}$

\section{NE correlation}

It is well known that the resistivity of oxides strongly depends on the oxygen stoichiometry. The migration/ diffusion of the oxygen-related defects, therefore, is an obvious candidate for the switching mechanism. ${ }^{3,5}$ As previously discussed, however, such models may have difficulty to ac- 


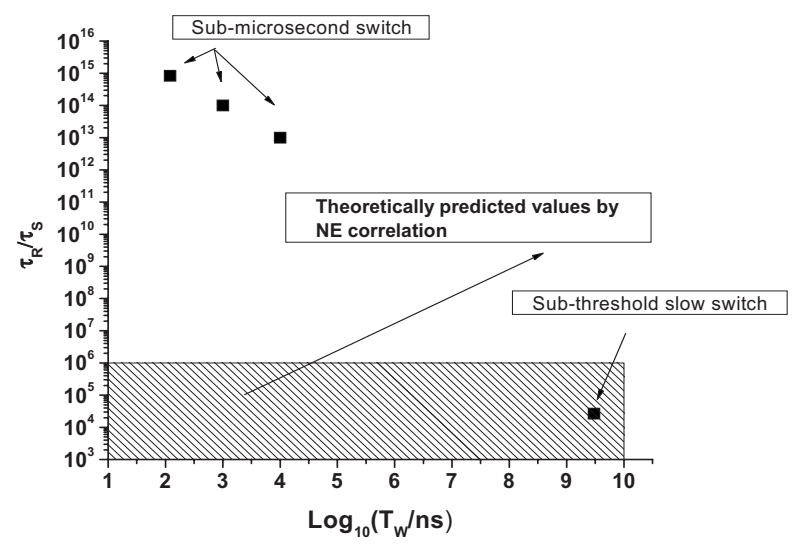

FIG. 11. Experimental verification of NE correlation for different types of switch in metal-PCMO interface. The first three points are sub- $\mu$ s fast switching $\left(T_{\mathrm{w}}=100 \mathrm{~ns}, 1 \mu \mathrm{s}\right.$, and $10 \mu \mathrm{s}$, respectively), and the last point (inside the shaded region explained by NE correlation) is a subthreshold slow quasistatic switch with $3 \mathrm{~s}$ pulse. $\tau_{\mathrm{R}}$ and $\tau_{\mathrm{S}}$ represent retention decay time constant and characteristic time scale for switching, respectively.

commodate the NE correlation in the case of sub- $\mu$ s switching. ${ }^{7}$ This correlation directly relates the defect migration rate under field with its thermal diffusion rate based on the assumption that both can be simplified as the same random-walk process of the pre-existing defects across the lattice (as an array of barriers); a reasonable assumption if the energy gain during each walk step is low enough to avoid irreversible changes in the lattice. The two rates, therefore, differ merely on the driving forces: concentration gradient, $k \mathrm{~T} \Delta n / \Delta x$ in diffusion but an effective $n Z e E$ in electromigration, where $n, \Delta n / \Delta x, e, E$, and $Z$ are the defect density, its gradient, the electronic charge, the local electric field, and an effective charge number, respectively. ${ }^{25}$ The effective charge $Z$ is used to consider the effects of carrier impacts. As a rough estimation $Z=n_{\mathrm{c}} \sigma l$, where $n_{\mathrm{c}}, \sigma$, and $l$ are the carrier concentration, the cross section of the defects against the carrier transport, and the mean free path, respectively. ${ }^{26}$ The value of $Z$, therefore, can hardly be larger than 100 with $n_{\mathrm{c}}$ $\ll 10^{-1} / \AA^{3}, \sigma \approx 1 \AA^{2}$, and $l \ll 100 \AA$ in highly disordered PCMO. The diffusion flux $J_{\mathrm{D}}$ and the electromigration flux $J_{\mathrm{E}}$ across the interfacial layer of thickness $\Delta x$, therefore, are $D \cdot d n / d \mathrm{x} \approx D \cdot \Delta n / \Delta x$ and $D n Z e E /(k \mathrm{~T})$, respectively, where $D$ is the diffusion constant. The ratio $\tau_{\mathrm{R}} / \tau_{\mathrm{S}}=\frac{J_{E}}{J_{D}}=\frac{V_{F} Z e}{k \mathrm{~T}} \frac{n}{\Delta n}$, therefore, will be independent of both the $D$ and the $\Delta x$ and hardly larger than $10^{6}$, where $\tau_{\mathrm{R}}, \tau_{\mathrm{S}}$, and $V_{\mathrm{F}}(\geq E \Delta x)$ are the retention time period, the switching time, and the pulse voltage, respectively. To verify this argument, we have plotted the ratio $\tau_{\mathrm{R}} / \tau_{\mathrm{S}}$ for both the sub- $\mu$ s switching and that under subthreshold quasistatic switch (Fig. 11). The shaded region in Fig. 11 is the region where the ratio falls in accordance with NE correlation. It is clearly evident from the figure that only the subthreshold slow switch falls in that region, suggesting an accumulative process, such as diffusion, responsible for this switch. Regardless of the nature of the accumulated mechanism responsible (more complex than simple diffusion as suggested by Fig. 10), Fig. 11 conclusively rules out any possibility of a diffusion/accumulated mechanism for

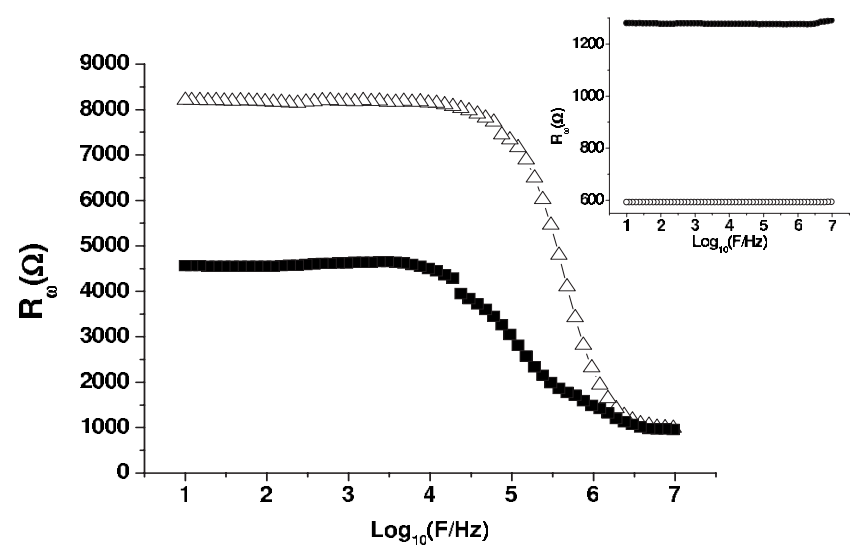

FIG. 12. (a) Frequency dependence ( $\log _{10} F$ on a linear scale) of interfacial resistance $R_{\omega}$ for fast above-threshold switch for $R_{\mathrm{HRS}}$ (solid squares) and $R_{\mathrm{LRS}}$ (open triangles). Inset: frequency dependence of interfacial resistance $\left(R_{\omega}\right)$ of the $R_{\mathrm{HRS}}$ (open circles) and $R_{\mathrm{LRS}}$ (solid circles) for subthreshold quasistatic slow sweep.

fast sub- $\mu$ s switch. This again reconfirms that mechanisms dominating the sub- $\mu$ s and the quasistatic switch are different in nature, as predicted earlier.

\section{Impedance spectroscopy and defects}

To further verify that the dominant mechanisms should be different for switches with sub- $\mu$ s pulses and those under quasistatic sweep, the impedance spectroscopy of electrodesample interfaces was used to extract the carrier trapping potentials. All samples used show a space charge-limited current (SCLC)-like I-V characteristic. Again, the three-lead configuration $^{10}$ was adopted and the complex $1 / \mathbf{Z}(\omega)$ $=\mathbf{I}(\omega) / \mathbf{V}(\omega)=1 / R(\omega)-i \omega \mathbf{C}$, where $\mathbf{Z}, \mathbf{I}$, and $\mathbf{V}$ are the impedance, the applied current, and the voltage drop, respectively, was used. As discussed earlier, ${ }^{7}$ this offers a way to characterize the trapping potentials of the defects responsible for switching in the SCLC region. A typical data set (sample C) plotting the frequency dependence of the interfacial resistance for fast switch (100 V, $1 \mu \mathrm{s}$ pulse), $R(\omega)\left[=R_{\omega}\right]$, is shown in Fig. 12. Strong dispersions appear around $100 \mathrm{kHz}$ (threshold frequency) as expected, suggesting either a mesoscopic inhomogeneity or the existence of defects with trapping potential $U$ on the order of $k \mathrm{~T} \ln \left(v / \omega_{0}\right)$ $(=0.30 \mathrm{eV}),{ }^{27,28}$ where $\omega_{0}$ is the threshold dispersion frequency. There has been much speculation in regard to the nature of the defects associated. This value does not support the oxygen diffusion model, as the $\mathrm{O}^{2-}$ vacancy defects are supposed to have a much smaller trapping potential. ${ }^{29-31}$

On the other hand, in the slow quasistatic de switch, no significant dispersion of $R_{\omega}$ was observed up to $10 \mathrm{MHz}$ for both HRS and LRS for sample B (inset, Fig. 12), a stark contrast to strong dispersions observed for high-voltage pulses invoking sub- $\mu$ s switching. This result reconfirms the existence of shallow point defects (trapping potential $\ll 0.2 \mathrm{eV}$ ) in the PCMO slow switch, which is different in nature from any of the deep traps/defect clusters discussed in fast switching. Although not conclusive, a cluster of point defects can produce deep trapping potentials that may be 
responsible for fast switching. Based on the above assumption, a field-induced local migration of ions/vacancies can cause a slow switch, whereas a rapid bond breaking and subsequent rearrangement driven by energy minimization can cause a sub- $\mu$ s fast switch. This also removes the ambiguity regarding the nature of slow switch (Fig. 10), suggesting an accumulative process but more complicated than a simple vacancy diffusion model.

\section{CONCLUSION}

The kinetics of switching and relaxation has been studied. The switching under narrow pulses up to $10 \mu$ s depends on the pulse amplitude with a steplike response. Above the threshold voltage $\left(V_{\mathrm{t}}\right)$, the switching is dominated by an exponential saturation of the $\Delta R$ with a time constant $\approx 200 \mathrm{~ns}$. The $\Delta R$ relaxation under such condition yields two separate regions: a relatively fast relaxation below 300 min followed by very steady metastable resistive states HRS and LRS. Both the initial amplitude and the time dependence of the fast-relaxation part are insensitive to the pulse width, suggesting a nonaccumulative process. The creation/ annihilation of lattice defects, such as bond breaking, and the relaxation of the distorted surrounding lattice, therefore, is suggested to be the mechanisms for the switching and the fast relaxation, respectively. Far below $V_{\mathrm{t}}$, on the other hand, the resistance hysteresis can only be invoked by quasistatic
I-V sweeps and/or pulses with widths longer than $10-10^{3} \mathrm{~s}$. The data suggest that such a process is largely accumulative but also that it is much more intriguing and cannot be explained by a simple migration/diffusion of the pre-existing defects.

However, during our work, a few queries came up that deserve sincere attention. Observations about the considerable deviation of relaxation from the diffusion equation for longer times $(>200 \mathrm{~min})$ and the upward relaxation directions toward a metastable state other than virgin state suggest the complex nature of the process. Further investigations are underway to fill in those fine details. Regardless, we believe our work provides a complete framework for a time-domain model for resistive switching, which is important from both a basic physics standpoint and an applications point of view.

\section{ACKNOWLEDGMENTS}

The work in Houston is supported in part by the U.S. Air Force Office of Scientific Research, the T. L. L. Temple Foundation, the John J. and Rebecca Moores Endowment, and the State of Texas through the Texas Center for Superconductivity at the University of Houston; and at Lawrence Berkeley Laboratory by the Director, Office of Science, Office of Basic Energy Sciences, Division of Materials Sciences and Engineering of the U.S. Department of Energy under Contract No. DE-AC03-76SF00098.
${ }^{1}$ M. J. Rozenberg, I. H. Inoue, and M. J. Sanchez, Phys. Rev. Lett. 92, 178302 (2004).

${ }^{2}$ S. Tsui, Y. Q. Wang, Y. Y. Xue, and C. W. Chu, Appl. Phys. Lett. 89, 123502 (2006).

${ }^{3}$ K. Szot, W. Speier, G. Bihlmayer, and R. Waser, Nature Mater. 5, 312 (2006)

${ }^{4}$ R. Dong, D. S. Lee, W. F. Xiang, S. J. Oh, D. J. Seong, S. H. Heo, H. J. Choi, M. J. Kwon, S. N. Seo, M. B. Pyun, M. Hasan, and H. Hwang, Appl. Phys. Lett. 90, 042107 (2007).

${ }^{5}$ Y. B. Nian, J. Strozier, N. J. Wu, X. Chen, and A. Ignatiev, Phys. Rev. Lett. 98, 146403 (2007).

${ }^{6} \mathrm{H}$. Song, M. Tokunaga, S. Imamori, Y. Tokunaga, and T. Tamegai, Phys. Rev. B 74, 052404 (2006).

${ }^{7}$ N. Das, S. Tsui, Y. Y. Xue, Y. Q. Wang, and C. W. Chu, Phys. Rev. B 78, 235418 (2008).

${ }^{8}$ S. H. Huerth, H. D. Hallen and B. Moeckly, Phys. Rev. B 67, 180506(R) (2003).

${ }^{9}$ B. Gautason and K. Muehlenbachs, Science 260, 518 (1993).

${ }^{10}$ A. Baikalov, Y. Q. Wang, B. Shen, B. Lorenz, S. Tsui, Y. Y. Sun, Y. Y. Xue, and C. W. Chu, Appl. Phys. Lett. 83, 957 (2003).

${ }^{11}$ Y. W. Xie, J. R. Sun, D. J. Wang, S. Liang, and B. G. Shen, J. Appl. Phys. 100, 033704 (2006).

${ }^{12}$ A. Odagawa, H. Sato, I. H. Inoue, H. Akoh, M. Kawasaki, Y. Tokura, T. Kanno, and H. Adachi, Phys. Rev. B 70, 224403 (2004).

${ }^{13}$ A. Sawa, T. Fujii, M. Kawasaki, and Y. Tokura, Appl. Phys. Lett. 85, 4073 (2004).

${ }^{14}$ R. S. Crandall, Phys. Rev. B 43, 4057 (1991).

${ }^{15}$ M. Stutzmann, Philos. Mag. B 56, 63 (1987).

${ }^{16}$ M. Stutzmann, Philos. Mag. B 60, 531 (1989).
${ }^{17}$ J. Bendler, J. Fontanella, and M. Shlesinger, J. Phys.: Condens. Matter 19, 065121 (2007).

${ }^{18}$ E. Montroll and G. Weiss, J. Math. Phys. 6, 167 (1965).

${ }^{19}$ Y. F. Chen and S. F. Huang, Phys. Rev. B 44, 13775(R) (1991).

${ }^{20}$ M. E. Glicksman, Diffusion in Solids: Field Theory, Solid-State Principles, and Applications (Wiley, New York, 2000).

${ }^{21}$ H. M. Ito, Y. Ogura, and M. Tomisaki, J. Stat. Phys. 66, 563 (1992).

${ }^{22}$ N. J. Mott and R. W. Gruney, Electronic Processes in Ionic Crystals (Dover, New York, 1948).

${ }^{23}$ J. Kakalios, R. A. Street, and W. B. Jackson, Phys. Rev. Lett. 59, 1037 (1987).

${ }^{24}$ A. Chantre and D. Bois, Phys. Rev. B 31, 7979 (1985).

${ }^{25}$ R. Meyer, R. Waser, J. Helmbold, and G. Borchardt, Phys. Rev. Lett. 90, 105901 (2003).

${ }^{26}$ D. Kandel and E. Kaxiras, Phys. Rev. Lett. 76, 1114 (1996).

${ }^{27}$ For a general review on dielectric relaxation, A. K. Jonscher, J. Phys. D 32, R57 (1999).

${ }^{28}$ J. J. Liu, C. Duan, W. N. Mei, R. W. Smith, and J. R. Hardy, J. Appl. Phys. 98, 093703 (2005).

${ }^{29}$ S. Mercone, A. Wahl, A. Pautrat, M. Pollet, and C. Simon, Phys. Rev. B 69, 174433 (2004).

${ }^{30}$ J. Sichelschmidt, M. Paraskevopoulos, M. Brando, R. Wehn, D. Ivannikov, F. Mayr, K. Pucher, J. Hemberger, A. Pimenov, H.-A. Krug von Nidda, P. Lunkenheimer, V. Yu. Ivanov, A. A. Mukhin, A. M. Balbashov, and A. Loidl, Eur. Phys. J. B 20, 7 (2001).

${ }^{31}$ N. A. Tulina, A. M. Ionov, and A. N. Chaika, Physica C 366, 23 (2001). 